\title{
Diagnosis of Giardia infections by PCR-based methods in children of an endemic area
}

David EB (1), Coradi ST (2), Oliveira-Sequeira TCG (1), Ribolla PEM (1), Katagiri S (1), Guimarães S (1)

(1) Department of Parasitology, Botucatu Biosciences Institute, São Paulo State University (UNESP - Univ Estadual Paulista), Botucatu, São Paulo State, Brazil; (2) Department of Biological and Health Sciences, Sacred Heart University, USC, Bauru, São Paulo State, Brazil.

\begin{abstract}
The present study was designed to estimate the prevalence of Giardia infection in preschool-and school-aged children living in an endemic area. Fecal samples from 573 children were processed by zinc sulfate centrifugal flotation, centrifugal sedimentation (using a commercial device for fecal concentration - TF-Test kit $^{\oplus}$ ) and polymerase chain reaction (PCR)-based methods. Of the stool samples assessed, 277 (48.3\%) were positive for intestinal parasites and/or commensal protozoa. Centrifugal flotation presented the highest diagnostic sensitivity for Giardia infections. The kappa index revealed that both coproparasitological techniques closely agreed on the Giardia diagnosis (86\%) versus satisfactory (72\%) and poor (35\%) concordances for commensal protozoan and helminth infections, respectively. Concerning Giardia molecular diagnosis, from the 71 microscopy-positive samples, specific amplification of $g d h$ and tpi fragments was noted in 68 (95.7\%) and 64 (90\%) samples, respectively. Amplification of $g d h$ and tpi genes was observed, respectively, in $95.7 \%$ and $90 \%$ of microscopy-positive Giardia samples. For 144 microscopynegative samples, $g d h$ and tpi gene amplification products were obtained from $8.3 \%$ and $35.9 \%$ samples, respectively. The agreement between these genes was about $40 \%$. The centrifuge-flotation based method was the most suitable means of Giardia diagnosis assessed in the present study by combining accuracy and low cost.
\end{abstract}

Key words: Giardia duodenalis, children, diagnosis, PCR.

\section{INTRODUCTION}

The protozoan Giardia duodenalis (syn. Giardia lamblia and Giardia intestinalis) stands out as the most frequent enteroparasite found in coproparasitological surveys conducted in developed and developing countries. In different populations, giardiasis is one of the most common nonviral causes of diarrhea among children, which, in turn, gives rise to such problems as malabsorption and weight loss, leading to delayed growth and development $(1,2)$.

Until today, the Giardia infection has usually been diagnosed under light microscopy to identify either trophozoites or cysts in fecal samples. In general, the examination of Giardia is performed in a single stool sample; however, as this parasite presents a variable pattern of excretion, misdiagnoses have been common and the actual prevalence may be underestimated. In view of this limitation, repeated samplings may be necessary, preferably a minimum of three samples on three alternate days. Concentration techniques such as formalin-ether or zinc sulfate flotation have been used routinely to diagnose this infection, but, even under ideal conditions, cysts are identified in a single stool sample in only $50-70 \%$ of samples (3). Commercially available concentration assays have been included in the laboratory routine to improve the coprological 
diagnosis, but they still show some limitations. In Brazil, the $\mathrm{TF}^{-T e s t}{ }^{\bullet}$ (Immunoassay, Brazil) is a newly available commercial kit designed for the diagnosis of human intestinal parasites in which the three collected fecal samples are pooled, double filtered and concentrated by centrifugation (4).

Despite the wide use of microscopic examination of stool in epidemiological surveys, the actual prevalence of Giardia infection may be underestimated in the communities. Therefore, the need for alternative methods has led some researchers to assess new procedures and techniques that can be efficient for this purpose. Recently, polymerase chain reaction (PCR)based methods that present sensitivity better than or similar to microscopy for directly detecting Giardia in stool have also been described (5). In this context, the present study was designed to estimate the prevalence of Giardia infection in children at preschool and school ages in an endemic area, using as diagnostic procedures the microscopy of fecal samples processed by centrifuge-flotation and by TF test assay and the PCR-based methods.

\section{MATERIALS AND METHODS}

\section{Study Population and Sample Collection}

From September 2007 to April 2009, fecal samples were obtained from 573 children aged zero to 14 years in the city of Pratânia, São Paulo state, Brazil. The study was approved by the Research Ethics Committee of the Botucatu Medical School, UNESP, under the protocol number 492/2009 CEPE.

For fecal sample collection, each child received a package containing three TF-Test kit ${ }^{\oplus}$ (Immunoassay, Brazil) collection tubes (patient kit) filled with a solution of potassium dichromate (2.5\%). This kit allowed the separate collection of three fecal specimens on alternate days.

\section{Coproparasitological Analysis}

Prior to the processing of samples, the three TF-Test kit ${ }^{\oplus}$ (Immunoassay, Brazil) collection tubes were vigorously agitated to achieve homogenization; from each sample, a part of the fecal material was transferred to a test tube. After this, the collection tubes were processed according to the TF-Test kit ${ }^{\circledast}$ manufacturer's instructions.
The fecal material transferred to the test tubes was washed three times with water $(350 \mathrm{~g}$ for one minute) to remove potassium dichromate. After the washes, an aliquot of fecal sediment was submitted to concentration analysis by the conventional coprological technique of centrifuge flotation in zinc sulfate, according to the method of Sloss et al. (6). The remaining sediment was used to prepare smears stained by a modified Ziehl-Neelsen (7) for detecting Cryptosporidium spp. and for DNA extraction.

Slides loaded with the material obtained by either TF-Test $\mathrm{kit}^{\oplus}$ or centrifuge-flotation in zinc sulfate were examined under an optical microscope to screen for the presence of Giardia and other intestinal parasites.

\section{DNA Extraction}

Total DNA was extracted from each Giardiapositive sample using the QIAamp ${ }^{\varpi}$ Stool mini kit (Qiagen, Germany) following the manufacturer's instructions. To optimize disruption of the cysts, prior to DNA extraction, the samples were subjected to three cycles of freezing and thawing by the following steps: two cycles alternating incubation in liquid nitrogen for five minutes and thawing in a water bath at $70^{\circ} \mathrm{C}$ for five minutes and concluding with a cycle of freezing in liquid nitrogen for five minutes and thawing at $95^{\circ} \mathrm{C}$ for five minutes.

Considering the possibility of false-negative results by coprological methods, Giardia-negative samples were processed for DNA extraction. The number of negative samples was calculated based on the prevalence data of Giardia infection in children in the municipality of Pratânia.

\section{PCR Assays}

Molecular diagnosis of Giardia was performed using two loci, glutamate dehydrogenase $(g d h)$ and triose phosphate isomerase $(t p i)$ genes. The eluted DNA was submitted to a semi-nested procedure for amplification of a 432-bp region from the $g d h$ gene according to Read et al. (8). A nested PCR reaction for amplification of a 530-bp fragment from the tpi gene was performed using a protocol described by Sulaiman et al. (9). In each reaction, negative ( $\mathrm{mix}+$ water) and positive (DNA from axenic G. duodenalis trophozoites) controls were added. The PCR products were submitted to $1.5 \%$ agarose gel electrophoresis, stained with ethidium bromide and the gel image was recorded under transilluminator UV light. 


\section{Statistical Analysis}

The frequencies of each parasite detected by centrifuge-flotation and TF-Test kit ${ }^{\circledast}$ were compared by the chi-square test. The diagnostic sensitivities of the techniques were individually calculated for each parasite by using the formula:

$$
S=\frac{a}{a+c} \times 100
$$

where "a" represents the number of positive cases detected by the method and " $c$ " is the number of true positive cases. The kappa index $(k)$ was calculated to determine the agreement among diagnoses obtained from each of the three methods. It is calculated based on the observed and expected frequencies displayed diagonally on a square table of frequencies (10), as follows:

$$
K=\frac{P_{0}-P_{e}}{1-P_{e}}
$$

where " $\mathrm{P}_{\mathrm{o}}$ " and " $\mathrm{P}$ " represent the observed and expected percentages of agreement, respectively.

All the analyses were done using Excel and PopTools (Microsoft Co., USA).

\section{RESULTS}

Of the 573 stool samples assessed, 277 (48.3\%) were positive for at least one intestinal parasite and/ or commensal. The most frequent parasite detected was Cryptosporidium spp., found in 79 (13.8\%) stool samples. In 49 samples, Cryptosporidium was detected as single infection (62\%) and in 30 (38\%) it was found associated with a commensal or with another intestinal parasite.

With exception of Cryptosporidium spp., the frequency of the other observed intestinal parasites and commensal protozoans are summarized in Table 1 according to the coprological method used. The following parasites, with their respective prevalences, were found in the fecal samples: G. duodenalis (12.4\%), Enterobius vermicularis (1.9\%), Trichuris trichiura (1.4\%), Ascaris lumbricoides (0.5\%) and Hymenolepis nana $(0.2 \%)$. The intestinal commensals included: Entamoeba coli (12.9\%), Endolimax nana (4.0\%) and Blastocystis hominis (1.2\%).

Of 198 positive samples, 165 were diagnosed by centrifugal flotation and 154 by the TF-Test ${ }^{\oplus}$ kit. Most of the samples positive for Giardia (98.6\%), E. vermicularis $(81.8 \%)$ and E. coli $(81.1 \%)$ were detected by centrifugal flotation, whereas the TF-Test ${ }^{\oplus}$ kit detected most of E. nana (87\%) B. hominis $(85.7 \%)$ and T. trichiura (75\%) infections, but statistical analysis revealed that the frequency of each individual parasite or commensal was similar regardless of the diagnostic method employed $(\mathrm{P}>0.05)$.

In light of the low prevalence of some parasites and commensals, the analytical sensitivity

Table 1. Frequency of intestinal parasites and commensals detected by centrifugal flotation (CF) and TFTest kit ${ }^{\oplus}$ (TF) in fecal samples from 573 children in São Paulo state, Brazil

\begin{tabular}{c|c|c|c}
\hline \multirow{2}{*}{ Parasites } & TF-Test & CF & Overall frequencies \\
\cline { 2 - 4 } & $\mathbf{n}(\%)$ & $\mathbf{n}(\%)$ & $\mathbf{n}(\%)$ \\
\hline Blastocystis hominis & $6(1.0)$ & $3(0.5)$ & $7(1.2)$ \\
\hline Endolimax nana & $20(3.5)$ & $17(3.0)$ & $23(4.0)$ \\
\hline Entamoeba coli & $57(10.0)$ & $60(10.5)$ & $74(12.9)$ \\
\hline Giardia duodenalis & $56(9.8)$ & $70(12.2)$ & $71(12.4)$ \\
\hline Ascaris lumbricoides & $3(0.5)$ & $2(0.4)$ & $3(0.5)$ \\
\hline Enterobius vermicularis & $6(1.0)$ & $9(1.6)$ & $11(1.9)$ \\
\hline Hymenolepis nana & $0(0)$ & $1(0.2)$ & $1(0.2)$ \\
\hline Trichuris trichiura & $6(1.0)$ & $3(0.5)$ & $8(1.4)$ \\
\hline
\end{tabular}


of each coprological method and the Kappa index of agreement (Table 2) were individually calculated only for Giardia, while for the others, these measures were calculated considering two groups: intestinal helminths (A. lumbricoides, T. trichiura and E. vermicularis) and intestinal commensals (E. coli, E. nana and B. hominis). Calculations revealed that centrifugal flotation provided the highest diagnostic sensitivity only for Giardia $(\mathrm{P}<0.01)$. The kappa Index revealed that coproparasitological techniques provided an excellent level of agreement in the diagnosis of Giardia (86\%) but satisfactory (72\%) and poor
(35\%) concordances for commensal protozoan and helminth infections, respectively.

As to Giardia molecular diagnosis, out of the 71 microscopy-positive samples, specific amplification of $g d h$ and tpi fragments was observed in 68 (95.7\%) and 64 (90.0\%) samples, respectively (Table 3 ). Not all of these samples showed PCR products for both genes. Of the 144 Giardia-negative samples by microscopy that were submitted to DNA amplification, PCR products of the expected size were generated in $12(8.3 \%)$ and $44(35.9 \%)$ samples, respectively for $g d h$ and tpi genes (Table 3 ).

Table 2. Sensitivity and agreement (kappa index) analyses of centrifuge-flotation (CF) and TF-Test ${ }^{\circledR}$ (TF) techniques utilized to diagnose intestinal parasites in 573 children in São Paulo state, Brazil

\begin{tabular}{c|c|c|c}
\hline \multirow{2}{*}{ Parasites } & \multicolumn{2}{|c|}{ Sensitivity } & \multirow{2}{*}{ CF x TF (kappa) } \\
\cline { 2 - 4 } & CF (\%) & TF (\%) & $0.86(0.79-0.93)$ \\
\hline Giardia duodenalis & $99^{*}$ & 79 & $0.35(0.02-0.70)$ \\
\hline Helminths & 65 & 65 & $0.72(0.64-0.81)$ \\
\hline
\end{tabular}

${ }^{*} p<0.01$

Table 3. Amplification of $g d h$ and tpi Giardia genes from 215 stool samples, microscopically positive (71) and negative (144), obtained from children in São Paulo state, Brazil

\begin{tabular}{c|c|c|c|c}
\hline \multirow{2}{*}{ Stool Exam } & \multicolumn{2}{|c|}{$g d h$} & \multicolumn{2}{c}{ tpi } \\
\cline { 2 - 5 } & Positive & Negative & Positive & Negative \\
\cline { 2 - 5 } & $\mathrm{n}(\%)$ & $\mathrm{n}(\%)$ & $\mathbf{n}(\%)$ & $\mathbf{n}(\%)$ \\
\hline Positive $(\mathrm{n}=71)$ & $68(95.7)$ & $03(4.3)$ & $64(90.0)$ & $07(10)$ \\
\hline Negative $(\mathrm{n}=144)$ & $12(8.3)$ & $132(91.7)$ & $44(35.9)$ & $100(64.1)$ \\
\hline Total $(\mathbf{n}=\mathbf{2 1 5})$ & $80(37.2)$ & $135(62.8)$ & $108(50.2)$ & $107(49.8)$ \\
\hline
\end{tabular}

Table 4. Agreement analysis (kappa index) between microscopic and molecular methods used for the diagnosis of Giardia infections

\begin{tabular}{c|c|c|c|c}
\hline Parasites & Mic $\times g d h$ & Mic x tpi & gdh $\times$ tpi & Mic x Mol \\
\hline Giardia duodenalis & 0.79 & 0.47 & 0.37 & 0.46 \\
\hline
\end{tabular}

Mic: microscopic diagnosis (CF and TF); Mol: molecular diagnosis ( $g d h$ and tpi). 
In relation to the agreement indexes (Table 4 ), the lowest concordance rate (47\%) was found by microscopic examination and PCR of the tpi gene. The $g d h$ gene results revealed a Kappa Index of 0.79 , which is interpreted as a satisfactory concordance (79\%). The agreement between the two genes was only $37 \%$. As to the correlation between microscopic examination (EM) and molecular techniques (TM) used, the $k$ value obtained (0.46) reflects a weak level of agreement.

\section{DISCUSSION}

The present study compared PCR-based methods with microscopy of concentrated fecal samples to assess their performance in diagnosing Giardia infection in children, during an epidemiological survey in an endemic area.

Giardia and other intestinal parasite infections were detected in this population by comparing the coprological diagnostic methods of centrifugal flotation and the TF-Test ${ }^{\circledR}$ assay. Centrifugal flotation provided higher diagnostic sensitivity for Giardia infections than the TF-Test ${ }^{\oplus}$ kit, a difference probably related to the principle of each method. Given that the centrifugal flotation technique is based on measuring the concentration by flotation, it is suitable for detecting lighter parasitic forms such as protozoan cysts. Despite the difference in their sensitivity for detecting Giardia, both techniques enabled the diagnosis of intestinal protozoa in the population with a high level of concordance, namely, $86 \%$ for Giardia and $72 \%$ for the commensal protozoans. The low prevalence of intestinal helminths in this population precludes further discussion on the reliability of these two methods.

As to the molecular diagnosis of Giardia infection, not all positive stool samples showed PCR products for $g d h$ and tpi. Even though molecular techniques offer advantages over conventional methods due to their higher sensitivity and specificity, problems in PCR performance have been reported by other researchers in Giardia studies (11-15). There is a consensus among the authors that the occurrence of false-negative cases is associated with factors that may determine the absence or low concentrations of DNA in the sample to be submitted to extraction and amplification of genetic material.
The presence of inhibitors in feces appears to be one of the most main factors that affect the efficiency of PCR-based techniques, resulting in non-amplification of the gene fragments. Among these inhibitors we highlight substances such as the polysaccharide complex, bile salts, bilirubins and hemoglobin degradation products (16). Besides the diversity of inhibitors in feces, the concentrations of these substances in the sample varies according to the stool, diet, gut flora and health condition of the host (16). To minimize the effect of DNA inhibitors, one alternative is using commercial kits that include extraction columns (spin columns) for purification of DNA. The extraction by these kits can reduce the amount of PCR inhibitors, as well as enable greater efficiency in obtaining the DNA sample. In the present study, despite using such a kit, the occurrence of false negatives was not eliminated and, thus, the amplification of fragments corresponding to $g d h$ and tpi genes was observed respectively in $95.7 \%$ and $90 \%$ of the samples. A similar situation has been found in recent studies in which the authors report target-fragment amplification in only $70 \%$ of Giardia-positive samples $(11,12)$.

In addition to PCR inhibitors, the low number of cysts in the stool can directly affect the concentration of the extracted DNA sample. With respect to Giardia, as the excretion of cysts is intermittent, cysts are not passed or are passed sporadically during a period of 15 to 20 days. The examination of at least three stool specimens collected on alternate days can be an alternative for minimizing the interference of this biological factor, but many times it does not avoid the problem when cysts are passed in very low numbers. Although this alternative has been adopted in the present study, it was not sufficient to eliminate the false-negative results.

Besides the limitations due the low concentration of cysts in feces, we may infer that the lysis of these forms is also a determining factor for obtaining the genomic DNA. Thus, to ensure a better yield in obtaining DNA, samples were subjected to a heat shock procedure which consists of alternating cycles of freezing in liquid nitrogen $\left(-196^{\circ} \mathrm{C}\right)$ and thawing in a water bath at temperatures up to $95^{\circ} \mathrm{C}$. This procedure produces satisfactory results, but it does not mean that $100 \%$ of cysts can be disrupted. The extent to which the goal of total cyst disruption is achieved depends on the number of cycles, temperature 
for thawing, duration of each cycle and also the number of cysts present in the sample.

The high sensitivity of PCR-based techniques requires attention in relation to the increased possibility of false-positive results. In 144 microscopy-negative samples, amplification products for the $g d h$ and $t p i$ genes were obtained from $8.3 \%$ and $35.9 \%$ of samples, respectively. Given that the occurrence of false-positives is frequently related to contamination by amplicons, preventive measures were adopted. Thus, the false-positive cases observed in our study could be attributed to the low concentration of cysts in stool samples, which caused the infection to be underestimated by microscopic examination.

With regard to amplification of DNA fragments by $g d h$ and tpi, the correlation between these genes was about $40 \%$, since not all samples were amplified by both molecular markers. Of the 214 DNA samples, amplification of $g d h$ and tpi gene fragments was observed respectively in $80(37.2 \%)$ and $108(50.2 \%)$ samples, while only in 61 samples $(28.5 \%)$ was it possible to obtain amplification products of fragments of both genes. Our observations corroborate recent studies in which the authors observed differences in the performance of commonly used markers of $g d h, t p i$ and $18 \mathrm{~S}$ rRNA genes in PCR amplification $(12,14)$. The cause of this difference is not yet known, but according to Lalle et al. (14), despite the fact that gene primers are designed to bind "conserved" regions in genes, it is possible that mismatches in primer sequences may be too long to allow successful PCR analysis of some fecal isolates.

Although molecular PCR has clearly enabled further improvements in parasite diagnosis and epidemiology, the successful application of PCR-based methods in epidemiological surveys depends on understanding the limitations and assumptions of the techniques. According to the present observations, the centrifugal flotationbased method remains the most suitable for Giardia diagnosis. Indeed, given that most endemic areas are distributed in developing countries, the diagnostic methods employed during an epidemiological survey should combine accuracy and low cost of the diagnosis.

\section{ACKNOWLEDGEMENTS}

We are grateful for the collaboration of Pratânia Municipal Administration. Special thanks are due to all the dedicated staff of Pratânia's Municipal Schools and of the day care center Angela Martin Basseto and to all children and their families for participating in this study.

\section{COPYRIGHT \\ (C) CEVAP 2011}

\section{SUBMISSION STATUS}

Received: February 2, 2011.

Accepted: April 15, 2011.

Abstract published online: April 18, 2011.

Full paper published online: May 31, 2011.

\section{CONFLICTS OF INTEREST}

There is no conflict.

\section{FINANCIAL SOURCE}

The State of São Paulo Research Foundation (FAPESP) provided the financial grants (process number 06/56151-3).

\section{ETHICS COMMITTEE APPROVAL}

The present study was approved by the Research Ethics Committee of Botucatu Medical School, UNESP, under the protocol number 492/2009 CEPE.

\section{CORRESPONDENCE TO}

SEMÍRAMIS GUIMARÃES, Departamento de Parasitologia, Instituto de Biociências, UNESP, Botucatu, SP, 18618-000, Brasil. Phone: +55 14 3811-6239. Email: sgviana@ibb.unesp.br.

\section{REFERENCES}

1. Savioli L, Smith H, Thompson RCA. Giardia and Cryptosporidium join the 'Neglected Diseases Initiative'. Trends Parasitol. 2006;22(5):203-8.

2. Farthing MJ, Mata L, Urrutia JJ, Kronmal RA. Natural history of Giardia infection of infants and children in rural Guatemala and its impact on physical growth. Am J Clin Nutr. 1986;43(3):395-405.

3. Mank TG, Zaat JO, Deelder AM, Van Eijk JT, Polderman AM. Sensitivity of microscopy versus enzyme immunoassay in the laboratory diagnosis of giardiasis. Eur J Clin Microbiol Infect Dis. 1997;16(8):615-9.

4. Gomes JF, Hoshino-Shimizu S, Dias LC, Araújo AJ, Castilho VL, Neves FA. Evaluation of a novel kit (TFTest) for the diagnosis of intestinal parasitic infections. J Clin Lab Anal. 2004;18(2):132-8.

5. Verweij JJ, Blangé RA, Templeton K, Schinkel J, Brienen EA, van Rooyen MA, et al. Simultaneous detection of Entamoeba histolytica, Giardia lamblia, and Cryptosporidium parvum in fecal samples by 
using multiplex real-time PCR. J Clin Microbiol. 2004;42(3):1220-3.

6. Sloss MW, Zajac AN, Kemp, RL. Parasitologia Clínica Veterinária. 6 ed. São Paulo: Manole; 1999. 198 p.

7. Henriksen A, Pohlenz JFL. Staining of cryptosporidia by a modified Ziehl-Neelsen technique. Acta Vet Scand. 1981;22(3-4):594-6.

8. Read CM, Monis PT, Thompson RCA. Discrimination of all genotypes of Giardia duodenalis at the glutamate dehydrogenase locus using PCR-RFLP. Infect Genet Evol. 2004;4(2):125-30.

9. Sulaiman IM, Fayer R, Bern C, Gilman RH, Trout JM, Schantz PM, et al. Triosephosphate isomerase gene characterization and potential zoonotic transmission of Giardia duodenalis. Emerg Infect Dis. 2003;9(11):1444-52.

10. Atman, S.D.C. Pratical statistics for medical research. Chapman and Hall. London, 1991. 611 p.

11. Volotão AC, Costa-Macedo LM, Haddad FSM, Brandão A, Peralta JM, Fernades O. Genotyping of Giardia duodenalis from human and animal samples from Brazil using $\beta$-giardin gene: a phylogenetic analysis. Acta Trop. 2007;102(1):258-62.
12. Gelanew T, Lalle M, Hailu A, Pozio E, Cacciò SM. Molecular characterization of human isolates of Giardia duodenalis from Ethiopia. Acta Trop. 2007;102(2): 92-9.

13. Lebbad M, Ankarklev J, Tellez A, Leiva B, Andersson JO, Svärd S. Dominance of Giardia assemblage B in León, Nicaragua. Acta Trop. 2008;106(1):44-53.

14. Lalle M, Bruschi F, Castagna B, Campa M, Pozio E, Cacciò SM. High genetic polymorphism among Giardia duodenalis isolates from Sahrawi children. Trans R Soc Trop Med Hyg. 2009;103(8):834-8.

15. Mohammed Mahdy AK, Surin J, Wan KL, MohdAdam A, Al-Mekhlafi MSH, Lim YAL. Giardia intestinalis genotypes: Risk factors and correlation with clinical symptoms. Acta Trop. 2009;112(1):67-70.

16. Gonçalves EM, Araújo RS, Orban M, Matté GR, Matté $\mathrm{MH}$, Corbett CEP. Protocol for DNA extraction of Cryptosporidium spp. Oocysts in fecal samples. Rev Inst Med Trop São Paulo. 2008;50(3):165-7. 